\title{
Research Article \\ Hollow Gold Nanoparticles Loaded with L-Buthionine- Sulfoximine as a Novel Nanomedicine for In Vitro Cancer Cell Therapy
}

\author{
Min Liu, ${ }^{1}$ Wushan Li $\mathbb{D},{ }^{2}$ Ri Xu $\mathbb{D}^{1},{ }^{1}$ Xiaoyan Jiang $\mathbb{D}^{1},{ }^{1}$ and Anchang Liu $\mathbb{D}^{1}$ \\ ${ }^{1}$ Department of Pharmacy, Qilu Hospital of Shandong University, Jinan, 250012 Shandong, China \\ ${ }^{2}$ Jinan Maternity and Child Care Hospital Affiliated to Shandong First Medical University, Jinan, 250012 Shandong, China
}

Correspondence should be addressed to Anchang Liu; anchangliu03@163.com

Received 6 May 2021; Revised 2 August 2021; Accepted 7 August 2021; Published 18 August 2021

Academic Editor: Philip D. Rack

Copyright (๑) 2021 Min Liu et al. This is an open access article distributed under the Creative Commons Attribution License, which permits unrestricted use, distribution, and reproduction in any medium, provided the original work is properly cited.

\begin{abstract}
A novel nanomedicine, constructed by simultaneously binding L-buthionine-sulfoximine (BSO) and thiolated polyethylene glycol (PEG) to the surface of $100 \mathrm{~nm}$ hollow gold nanoparticles (HAuNS), was expected to be used in effective therapeutics of cancer. The current study is aimed at evaluating in vitro the antitumor efficacy of newly synthesized BSO-loaded PEG-SH-HAuNS (BSO@HAuNS) with strong resonances in near-infrared (NIR) as a chemotherapy agents against a line of human lung cancer cells (A549). Here, we conducted cytotoxicity assays and found BSO@HAuNS to efficiently kill human lung cancer cells by ROS generation, indicating that BSO facilitated an increased susceptibility of cancer cells to PEG-SH-HAuNS. Based on flow cytometry analysis, BSO@HAuNS can induce apoptosis and necrosis in mitochondrial-dependent pathway in A549 cells. Our results revealed a novel class of nanomedicine with high potential to be implemented as effective chemotherapy agents for patients diagnosed with unresectable lung cancer.
\end{abstract}

\section{Introduction}

Despite the advances made in early detection and treatment of tumor in the past decades, cancer remains one of the most difficult diseases to treat as well as a leading cause of morbidity and mortality [1]. For treating inoperable cancer, the limitations of the traditional chemotherapy are well known, necessitating the development of novel alternatives which overcome the poor specificity, side effects, or limited accessibility of currently employed methods.

To overcome problems with existing anticancer therapies, gold nanoparticles (GNPs) are being explored recently due to their ease of synthesis, high biocompatibility, tendency to accumulate in cancerous tissues by the enhanced permeability and retention (EPR) effect, and facile surface chemistry allowing conjugation to biologically active molecules [2-5]. GNPs are ideal as a Drug-Delivery Vector, which provides extraordinary opportunities that can offer both passive and active targeting abilities towards cancerous tissues/cells [6-8]. Currently, there are numerous reports that gold nanoparticles, when used as drug carriers for cancer treatment, yield more favorable results compared to standard anticancer drug treatments. For example, the combination of gold nanoparticles and doxorubicin (DOX) achieves significantly enhanced the cytotoxicity of doxorubicin and induces elevated apoptosis of cancer cells [9-11]. Nanocomposites made of platinum-functionalized gold nanoparticles exhibit higher cytotoxicity and increased efficacy than platinum alone $[4,12]$. The nanoparticle delivery system consisting of polyethylene glycol- (PEG-) coated colloidal gold nanoparticles with incorporated TNF- $\alpha$ payload was developed to maximize tumor damage and minimize systemic exposure to TNF- $\alpha$ [13]. Heo et al. [14] designed and prepared paclitaxel- (PTX-) conjugated gold nanoparticles that can result in the targeted release of PTX inside tumor cells and increase tumor cell killing efficiency. Meanwhile, many researchers have reported that gold nanoparticles may be employed as potential therapeutics by altering intracellular redox status in cancer cells [15]. Previously, we found that the cytotoxicity of gold nanoparticles is 
associated with intracellular glutathione (GSH) levels [16]. We also demonstrated that gold nanoparticles $(10 \mathrm{~nm})$ can trigger apoptosis and necrosis in human lung cancer cells (A549) when L-buthionine-sulfoximine (BSO) was used to decrease the expression of intracellular glutathione [17]. In the present work, we further hypothesized that loading of BSO on GNP will have a synergetic effect against human lung cancer cells.

Compared to solid core GNPs, hollow gold nanoparticles (HAuNS), a novel class of GNPs composed of a thin gold shell with a hollow interior [18], exhibit much higher optical sensitivity and their strong, tunable absorption band suggests that HAuNS are a promising mediator for drug delivery in cancer treatment [19]. Furthermore, hollow gold nanoparticles with porous shell structure can best be used as carriers for therapeutic compounds in vivo. A few studies have explored the potential utility of HAuNS as a novel delivery vehicle to shuttle biomolecules $[20,21]$. In this study, we carried out HAuNS embedded with BSO to serve as a novel nanomedicine and evaluated the cytotoxity of hollow gold nanoparticles loaded with L-buthioninesulfoximine (BSO@HAuNS) towards A549 cells.

\section{Methods and Materials}

2.1. Materials. Silver nitrate $\left(\mathrm{AgNO}_{3}\right.$, A.R.), ethylene glycol (EG, A.R.), chloroauric acid ( $\mathrm{HAuCl}_{4} \bullet 4 \mathrm{H}_{2} \mathrm{O}$, A.R.), ascorbic acid (AA, A.R.), cetyltrimethylammonium bromide (CTAB, A.R.), and nitric acid $\left(\mathrm{HNO}_{3}, A . R.\right)$ were obtained from Shanghai Sinopharm Chemical Reagent Co. Ltd. Polyvinylpyrrolidone (PVP, $\mathrm{Mw} \approx 55000$ ) was obtained from SigmaAldrich. All chemicals were used as purchased without further purification. $\mathrm{HAuCl}_{4}$ aqueous solution $(1 \mathrm{mM})$ was prepared by dissolving $\mathrm{HAuCl}_{4} \bullet 4 \mathrm{H}_{2} \mathrm{O}$ in ultrapure water $(18.23 \mathrm{M})$. FITC-labeled Annexin V/PI apoptosis detection kit was obtained from Invitrogen-Life Technologies. Lbuthionine-sulfoximine (BSO) and polyethylene glycol were purchased from Sigma-Aldrich. 2,7-dichlorodihydro fluorescent diacetate (DCFH-DA) and Glutathione Quantification Kit were purchased from Beyotime Institute Biotechnology.

\subsection{Chemical Synthesis of Hollow Gold Nanoparticles} (HAuNS). Firstly, $\sim 100 \mathrm{~nm}$ silver nanoparticles were fabricated by a classic polyol process. Then, an electroless plating approach was employed to synthesize hollow gold nanospheres (HAuNS). In a typical process, a fixed amount $(0.02 \mathrm{M}, 1 \mathrm{~mL})$ of $\mathrm{Ag}$ nanoparticles was dispersed in $17 \mathrm{~mL}$ water containing $\sim 12 \mathrm{mM} \mathrm{CTAB}$ and $3 \mathrm{mM}$ ascorbic acid in a $25 \mathrm{~mL}$ vial under magnetic stirring and then heated in a water bath at $40^{\circ} \mathrm{C}$ for $10 \mathrm{~min} .2 \mathrm{~mL}$ of $1 \mathrm{mM} \mathrm{HAuCl}_{4}$ was added to the vial by droplet. The solution was heated for another $25 \mathrm{~min}$ until the color became stable. Then, the sample was centrifuged and washed with ultrapure water to remove excess $\mathrm{Cl}-$, $\mathrm{CTAB}$, and ascorbic acid before dealloying by nitric acid. The samples were redispersed in $5 \mathrm{~mL}$ ultrapure water, and a specific amount of nitric acid was added to the solution. After 15 min dealloying, the samples were centrifuged and washed with ultrapure water and
$\mathrm{NH}_{3} \bullet \mathrm{H}_{2} \mathrm{O}$ to remove excess nitric acid and trace amount of AgCl before characterization.

2.3. Preparation of BSO-Loaded PEG-SH-HAuNS. To synthesize thiolated polyethylene glycol-functionalized gold nanoparticles (PEG-SH-HAuNS), $1 \mathrm{mM}$ hollow gold nanoparticles were mixed with thiolated polyethylene glycol (PEGSH, MW: 5000, $100 \mu \mathrm{M}$ ) and stirred overnight. A low ratio was preferred to avoid encapsulation of nanoparticles with a thicker layer of PEG. Purification was carried out by ultracentrifugation $(12000 \times \mathrm{g})$ using water to remove the unreacted PEG and concentrate the PEGylated HAuNS solution for use in vitro experiments. Next, we modified the functionalized gold nanoparticles with BSO to fabricate a nanocarrier. $1 \mathrm{mM}$ BSO was added to a dispersion of PEGSH-HAuNS $(0.5 \mathrm{mM})$ obtained as described above. The solution was incubated for $24 \mathrm{~h}$ at room temperature and then centrifuged at $12000 \times \mathrm{g}$ for $0.5 \mathrm{~h}$. The pellets thus obtained after centrifugation were separated from the supernatant solution and redispersed in Milli Q water prior to further use.

2.4. Cell Culture. A549 cells were purchased from the Cell Bank of Typical Culture Collection of Chinese Academy of Sciences (Shanghai, China). The cells were maintained in RPMI 1640 medium consisting of $4.5 \mathrm{~g} / \mathrm{L}$ glucose, $2 \mathrm{mM} \mathrm{L}$-glutamine, $1 \mathrm{mM}$ sodium pyruvate, supplemented with $10 \%$ fetal bovine serum, $100 \mathrm{U} / \mathrm{mL}$ penicillin, and $100 \mathrm{mg} / \mathrm{mL}$ streptomycin at $37^{\circ} \mathrm{C}$ in a $5 \% \mathrm{CO}_{2}$ humidified incubator.

2.5. Determination of GSH. GSH was determined using a commercial kit based on the glutathione reductase reaction which yields a yellow colored 5-thio-2-nitrobenzoic acid according to the manufacturer's protocol. The samples (cell extracts after treated with or without BSO@HAuNS) were processed through exactly the same procedure. Glutathione contents were determined by $412 \mathrm{~nm}$ absorbance with a multiwell plate reader [16].

2.6. Cytotoxicity Assay of BSO-Loaded PEG-SH-HAuNS. Human lung cancer cells (A549) were chosen as a cancer cell model to evaluate the toxicity of BSO@HAuNS. Cells in log phase were trypsinized and seeded in 6-well plates. After $24 \mathrm{~h}$ incubation, the medium with different concentrations of PEG-SH-HAuNS or BSO@HAuNS was added into the 6-well plates, and the cells were cultured for additional $72 \mathrm{~h}$. In vitro cytotoxicity of BSO-loaded PEG-SH-HAuNS against lung cancer cells was evaluated via cell counting. After extraction of medium in well, cultures were treated with trypsin-EDTA, and the released cells were resuspended to a final volume of a $2 \mathrm{~mL}$ cell medium. The cell numbers in each sample were counted under a microscope using a Counting Chamber Set (Qiujing Inc).

2.7. Measurement of Intracellular Reactive Oxygen Species (ROS) Production. The amount of ROS was measured using the 2,7-dichlorodihydro fluorescent diacetate (DCFH-DA) assay as previously described [16]. A549 cells were seeded into 6-well plates; after recovery, cells were exposed to different treatments, with nontreated cells used as negative control. The treatment groups included BSO, PEG-SH- 
HAuNS, BSO@HAuNS, and BSO@HAuNS+GSH. At $72 \mathrm{~h}$ after treatment, the cells were washed with PBS for three times and subsequently treated with $10 \mu \mathrm{M}$ DCFH-DA for 30 minutes. Then, the cells were washed with PBS, trypsinized, and collected by centrifugation. Cells (50000) in $200 \mu \mathrm{L}$ PBS solution from each sample were transferred to a 96-well black microplate. The fluorescent signal was measured with a fluorescence spectrophotometer $(\lambda \mathrm{ex}=488 \mathrm{~nm}$ and $\lambda \mathrm{em}=525$ ).

2.8. Apoptosis and Necrosis Assay. To further quantitatively evaluate the suppression of BSO@HAuNS on A549 cells, apoptosis and necrosis were analyzed with the Annexin Vfluorescein isothiocyanate (FITC) apoptosis detection kit following the manufacturer's instructions. A549 cells were grown in 6-well plates and exposed for 72 hours to PEGSH-HAuNS conjugated or unconjugated with BSO. After the incubation period, cells were harvested by trypsin, washed with cold PBS, and centrifuged at $1000 \mathrm{rpm}$ for $5 \mathrm{~min}$. Cells were resuspended in $100 \mu \mathrm{L}$ binding buffer; Annexin V-FITC and PI were added to each sample. Samples were then incubated at room temperature in the dark for $15 \mathrm{~min}$ and analyzed by flow cytometry. For this technique, the cells that are negative to both PI and Annexin V staining are the alive ones, the cells PI-negative and Annexin $\mathrm{V}$-positive staining are considered to be early apoptotic ones, and the cells PI-positive and Annexin V-positive staining are those in the latest stages of apoptosis or necrosis [17].

\subsection{Measurement of Mitochondrial Membrane Potential.} Mitochondrial damage leads to dissipation of transmembrane potential increases. JC-1 (5,50,6,60-tetrachloro-1,10,3,30-tetraethylbenzamidazolocarbocyanin iodide) was used to detect mitochondrial depolarization occurring in the early stage of apoptosis. Mitochondrial membrane potential $(\triangle \Psi \mathrm{m})$ analysis was conducted and modified, as described previously [17]. Briefly, A549 cells were incubated with PEG-SH-HAuNS or BSO@HAuNS for $72 \mathrm{~h}$. Following incubation, cells were collected, incubated with JC-1 for 30 minutes in darkness at $37^{\circ} \mathrm{C}$. After staining, cells were centrifuged at $1000 \mathrm{rpm}$ for $5 \mathrm{~min}$, supernatant was removed, and the pellet was gently rinsed with PBS twice and then resuspended in PBS. The suspension was analyzed by flow cytometry.

2.10. Measurement of Intracellular $\mathrm{Ca}^{2+}$. Intracellular $\mathrm{Ca}^{2+}$ measurement was performed as described earlier [17]. After incubation with BSO@HAuNS, cells were collected, washed in PBS, and then loaded with Fluo-4/AM. The stained cells were incubated at $37^{\circ} \mathrm{C}$ for $40 \mathrm{~min}$ under protection from light and washed twice in PBS. The Fluo-4/AM-loaded cells were resuspended; then, $\mathrm{Ca}^{2+}$-dependent fluorescence intensity was measured in fluorescence channel FL-1 with an excitation wavelength of $488 \mathrm{~nm}$ and an emission wavelength of $530 \mathrm{~nm}$ in FACS analysis [17].

2.11. Measurement of Intracellular Cleaved Caspase-3. Active caspase-3 was detected by flow cytometry with cleaved caspase-3 (Asp175) antibody [17]. A549 cells in the logarithmic growth phase were plated in 6-well plates and incubated for $24 \mathrm{~h}$. Then, cells were treated with PEG-SH-HAuNS or
BSO@HAuNS for $72 \mathrm{~h}$. After treatment, cells were collected, fixed with $4 \%$ paraformaldehyde, treated with $0.1 \%$ Triton X-100, and blocked with $1 \%$ BSA. Then, cells were incubated with cleaved caspase-3 (Asp175) antibody (Alexa fluor 488 conjugate) for $30 \mathrm{~min}$, washed with PBS twice, and measured by flow cytometry.

2.12. Data Analysis. Data were expressed as means \pm standard deviation. Statistical significance values were obtained by one-way analysis of variance (ANOVA) with a 95\% confidence level using GraphPad Prism 5 statistics program. Significance was accepted at the level of ${ }^{*} p<0.05$ and ${ }^{* *} p<0.01$, and all experiments were performed in triplicate.

\section{Results and Discussion}

3.1. Characterization of Hollow Gold Nanoparticles. Hollow gold nanoparticles (HAuNS) were readily coated with thiolated polyethylene glycol. Functionalization of gold nanoparticles with PEG-SH was determined by transmission electron microscopy (TEM), UV-Vis spectrum. As shown in Figure 1(a), TEM analysis of prepared sample revealed the formation of nanoscale particles with nearly uniform size distribution. Mixing of PEG-SH with gold nanoparticles increased the negative charge of the particles which causes monodisperse and stable nanoparticles. The monodisperse PEG-SH-HAuNS with the average size of $100 \mathrm{~nm}$ were produced. The absorption spectra showed that the plasma resonance peak for PEG-SH-HAuNS was tuned to the NIR region $(\sim 1000 \mathrm{~nm})$ (Figure 1(b)).

3.2. Effect of BSO@HAuNS on Intracellular GSH. It is known that BSO can block the cell GSH expression by inhibiting $\gamma$ glutamyl cysteine synthetase ( $\gamma$-GCS) [22, 23]. Therefore, we modify the functionalized gold nanoparticles with BSO to fabricate a nanomedicine. Next, we measured the intracellular levels of GSH upon incubation in the presence or absence of BSO@HAuNS. As shown in Figure 2, 72 hours of incubation in the presence of BSO@HAuNS decreased the intracellular concentrations of GSH.

3.3. In Vitro Cytotoxicity. On the basis of cytotoxicity assay, gold nanoparticles have been found to be "nontoxic" or "toxic," depending on the physicochemical characteristics (surface charge, size, and surface chemistry) of nanoparticles [24]. Here, we analyzed the ability of the hollow gold nanoparticles (HAuNS) and BSO@HAuNS to induce cell death using cell counting assays with the A549 human lung epithelial cancer cell line. Cells were treated for $72 \mathrm{~h}$ with PEG-SHHAuNS or BSO@HAuNS, and the cell numbers were counted. The PEGylated hollow gold nanoparticles demonstrated no cytotoxicity at concentrations up to $100 \mu \mathrm{M}$ in A549 cell line (Figure 3(a)). In contrast, BSO@HAuNS significantly decreased the viability. As shown in Figure 3(b), compared to control, BSO@HAuNS obviously reduced A549 cell numbers whereas BSO or PEG-SH-HAuNS alone were not toxic to lung cancer cells. As a result, BSO and PEG-SH-HAuNS added together do have a synergistic effect on A549 cell inhibition. 


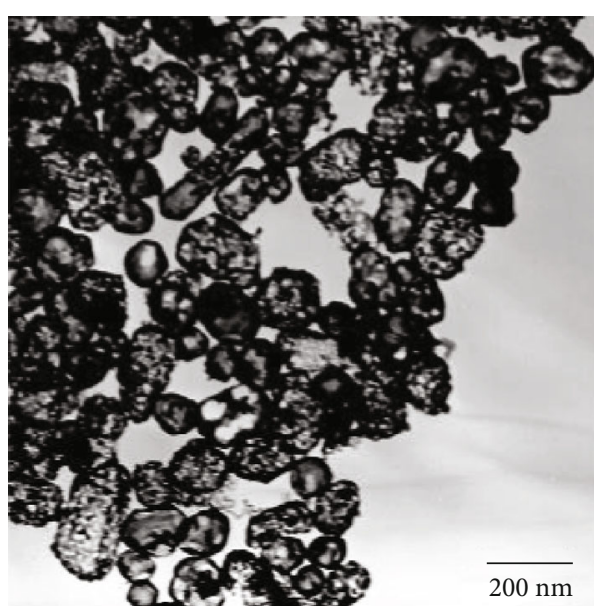

(a)

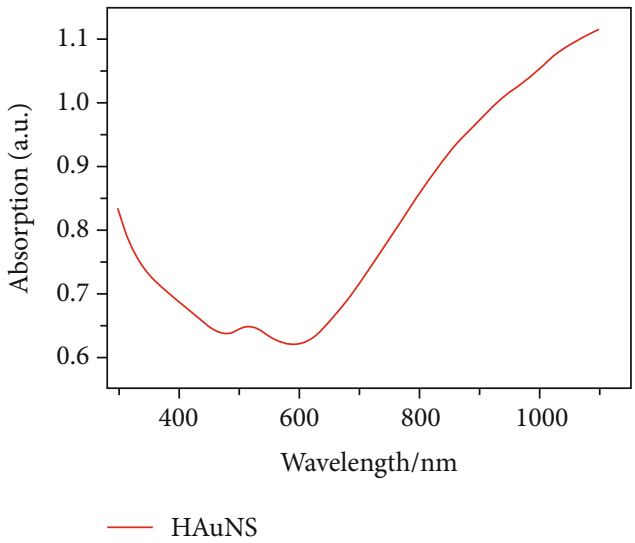

(b)

FIGURE 1: Characterization of the hollow gold nanoparticles (HAuNS). (a) A typical TEM image of the composite hollow gold nanoparticles. (b) UV-Vis absorption spectra showed characteristic peaks corresponding to surface plasmon of HAuNS.

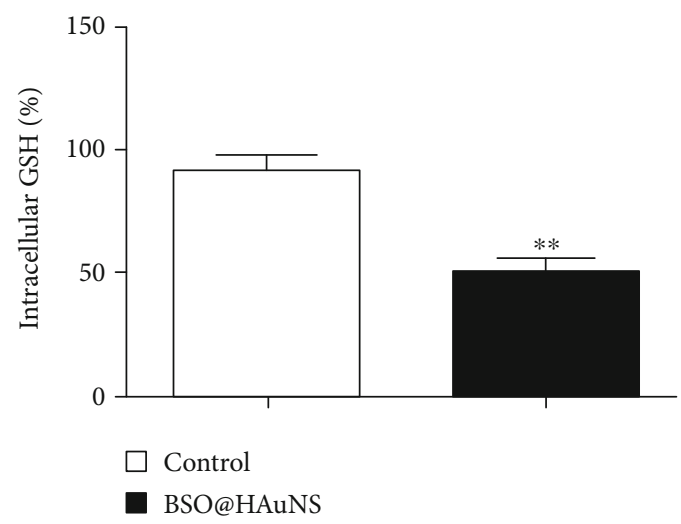

Figure 2: Influence of BSO-loaded PEG-SH-HAuNS on intracellular GSH levels. Arithmetic means $( \pm S D, n=3-4)$ of the GSH concentration of A549 cells following treatment for $72 \mathrm{~h}$ in $20 \mu \mathrm{M}$ BSO-loaded PEG-SH-HAuNS. ${ }^{* *} p<0.01$ indicates significant difference from control.

3.4. Measurement of Reactive Oxygen Species Production. It has been reported that nanoparticles can trigger a burst of reactive oxygen species (ROS) activity, thereby inducing a cascade of cytotoxic effects [25]. Previously, we showed that one of the primary mechanisms of cytotoxity of gold nanoparticles (GNPs) is generation of ROS [16]. To determine if the cytotoxic effect of the BSO@HAuNS in A549 cells could be attributed to oxidative stress, the ROS was measured using the DCFH-DA assay. The amount of ROS following treatment with BSO-loaded PEG-SH-HAuNS increased (Figure 4). The levels of ROS after treatment with the BSO@HAuNS in combination with antioxidants (GSH) were also evaluated. As shown in Figure 4, cotreatment of cells with antioxidants (GSH) and BSO@HAuNS resulted in ROS levels that were similar to those achieved following treatment with PEG-SH-HAuNS alone.

3.5. The Effect of BSO@HAuNS Treatment on Induction of Apoptosis and Necrosis in Lung Cancer Cells. Previous study reported that GNPs can induce apoptosis and necrosis in human lung cancer cells when BSO was used to decrease the intracellular GSH $[16,17]$. To determine whether cytotoxicity of BSO@HAuNS in A549 cells is also mediated by the same mechanism, apoptosis and necrosis of human lung cancer cells were examined by a double-staining method using FITC-labeled Annexin V/PI apoptosis detection kit and analyzed by flow cytometry. Typically, the cells were collected after 72 hours of exposure to a combination of PEG-SH-HAuNS loaded with BSO. Meanwhile, media containing floating cells were collected. All solutions were combined and centrifuged into pellets, which were redispersed in a binding buffer and treated with PI and Annexin $\mathrm{V}$. The percentage of apoptotic and necrotic cells (early apoptotic cells positive only for Annexin- $\mathrm{V}+$ and late apoptotic and necrotic cells positive for both Annexin $\mathrm{V}+$ and PI +) was found to increase (Figure 5).

3.6.BSO@HAuNS Alter Mitochondrial Membrane Potential in A549 Cells. Apoptosis and necrosis have been related to the disruption of mitochondrial membrane integrity, which 


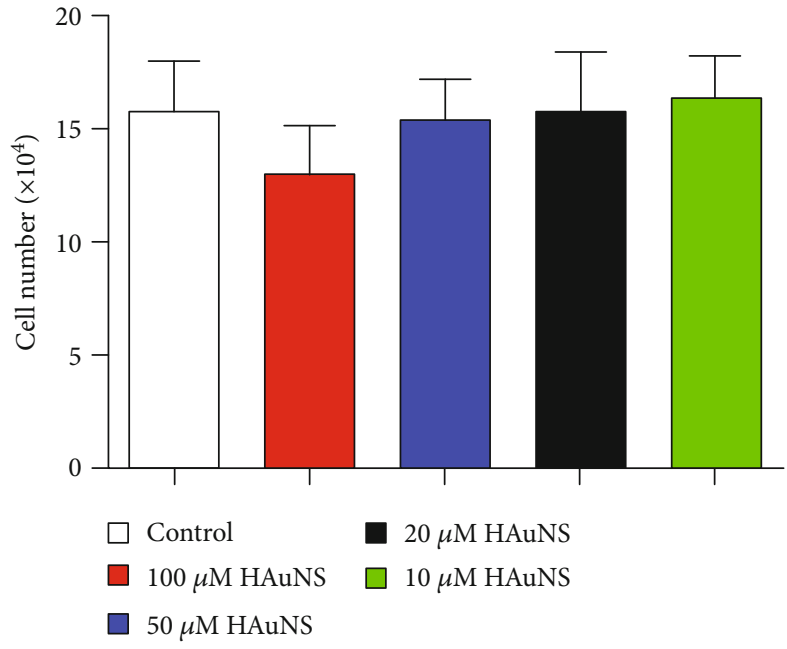

(a)

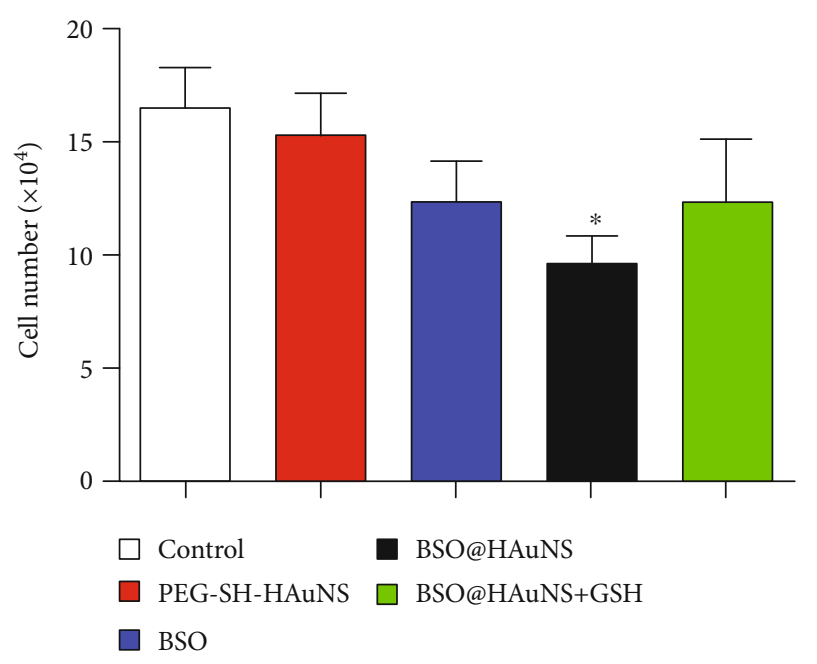

(b)

FIGURE 3: Differential cytotoxicity results for A549 cells exposed to HAuNS and BSO@HAuNS. (a) A549 cells were incubated with different concentrations HAuNS for $72 \mathrm{~h}$, subsequently harvested and counted. (b) A549 cells were treated with PEG-SH-HAuNS, BSO, and BSO@HAuNS for $72 \mathrm{~h}$. Viability was determined by cell counting. Data are representative of three independent experiments. ${ }^{*} p<0.05$ compared to control group.

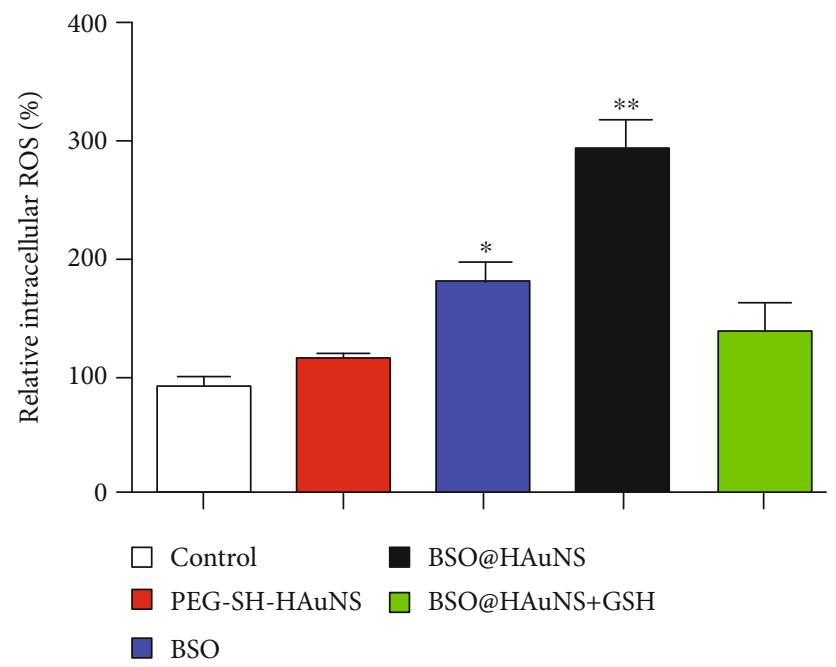

FIGURE 4: Amount of ROS produced relative to nontreated cells following treatment with BSO@HAuNS in combination with antioxidants including GSH. After different treatment, cells were harvested and counted. The levels of ROS in samples were measured using the DCFH$\mathrm{DA}$ assay. The mean number of control was taken as $100 \%$, and other values were calculated relative to this. Data represent mean $( \pm \mathrm{SD}$, $n=4) .{ }^{*} p<0.5$ and ${ }^{* *} p<0.01$ compared to control group.

is critical in the cell-death process [17]. The consequent loss of mitochondrial membrane potential $(\triangle \Psi \mathrm{m})$ is an early event in the initiation and activation of apoptotic and necrotic cascades [26]. Our group has reported that GNPs $(10 \mathrm{~nm})$ disrupt mitochondrial membrane potential in lung cancer cells with low intracellular GSH. Therefore, we further examine the depolarization of mitochondrial membrane by measuring the fluorescence remission shift (red to green) of the $\triangle \Psi \mathrm{m}$ sensitive cationic JC-1 dye in BSO@HAuNStreated cells. As shown in Figure 6(a), mitochondria did not depolarize after treatment with PEG-SH-HAuNS. Noteworthy was the observation that cells treated with BSO@HAuNS showed a decrease in red/green fluorescence intensity indicating increased mitochondrial membrane depolarization (Figure 6(a)). The result showed that BSO@HAuNS initiate apoptosis and necrosis in lung cancer cells by disrupting mitochondrial membrane integrity.

\subsection{BSO@HAuNS Evaluate the Cytosolic $\mathrm{Ca}^{2+}$ Concentration} in Lung Cancer Cells. Mitochondria can be considered as a firewall that controls the $\mathrm{Ca}^{2+}$ concentration in the cell and in cytoplasmic microdomains [27]. Our results above have shown that BSO@HAuNS disrupt mitochondrial function and induce apoptosis and necrosis. In the next experiment, Fluo4/AM fluorescence has been employed to estimate the cytosolic $\mathrm{Ca}^{2+}$ concentration. As illustrated in Figure 6(b), 

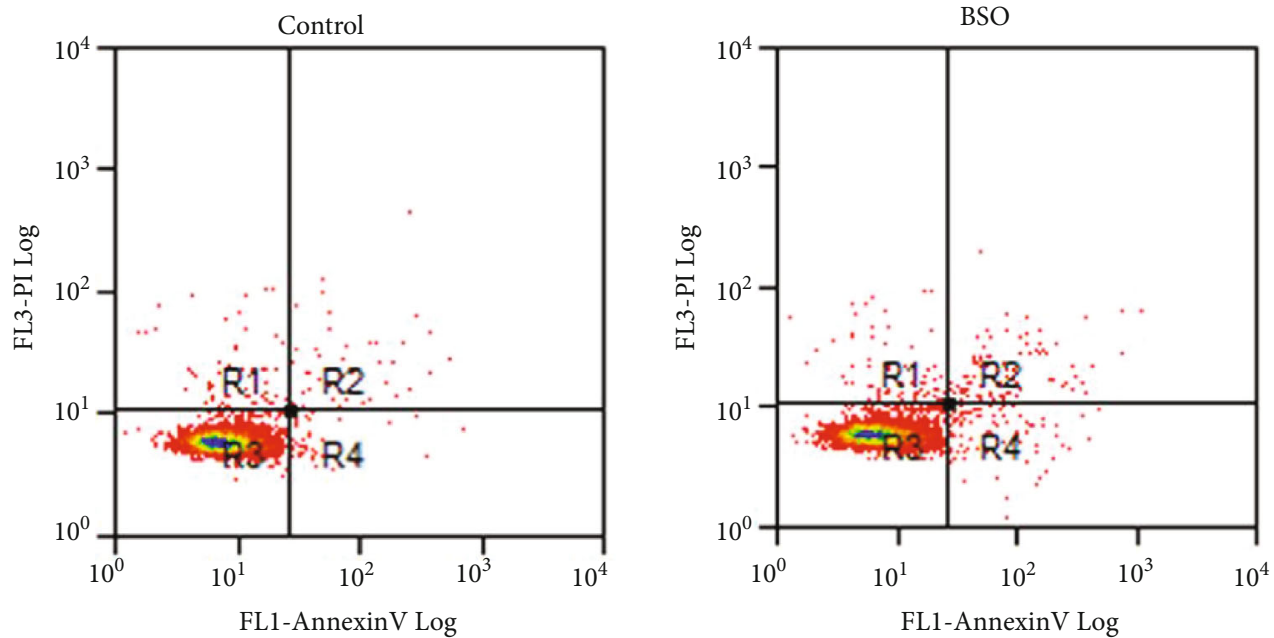

PEG-SH-HAuNS
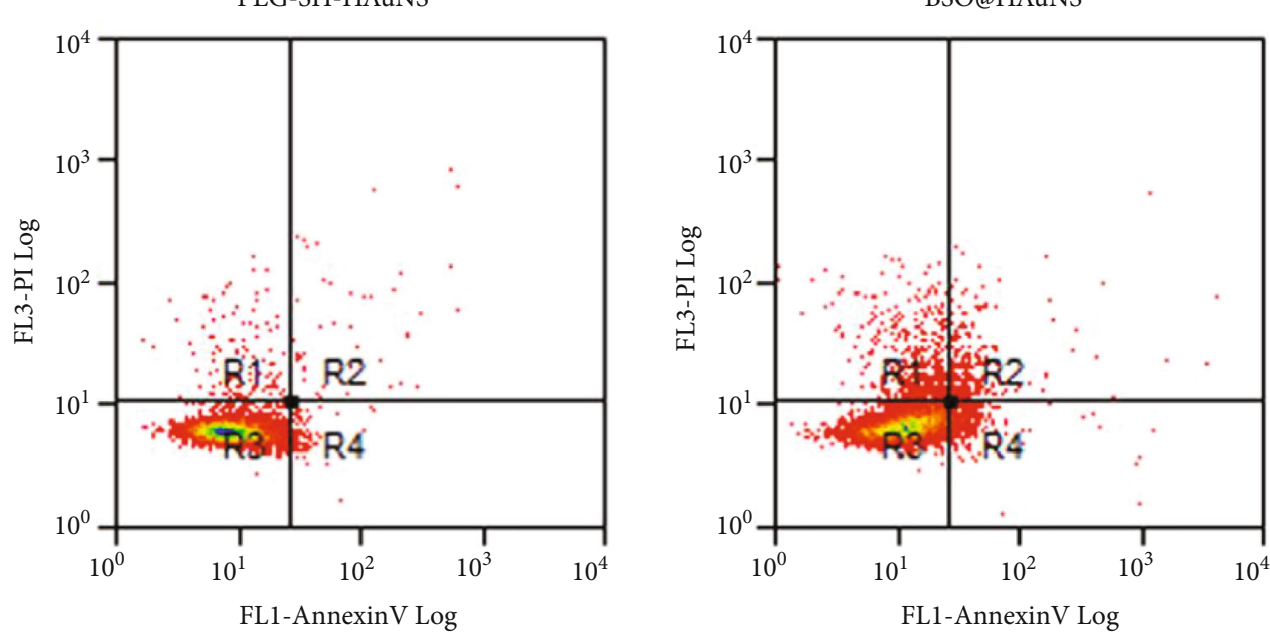

(a)
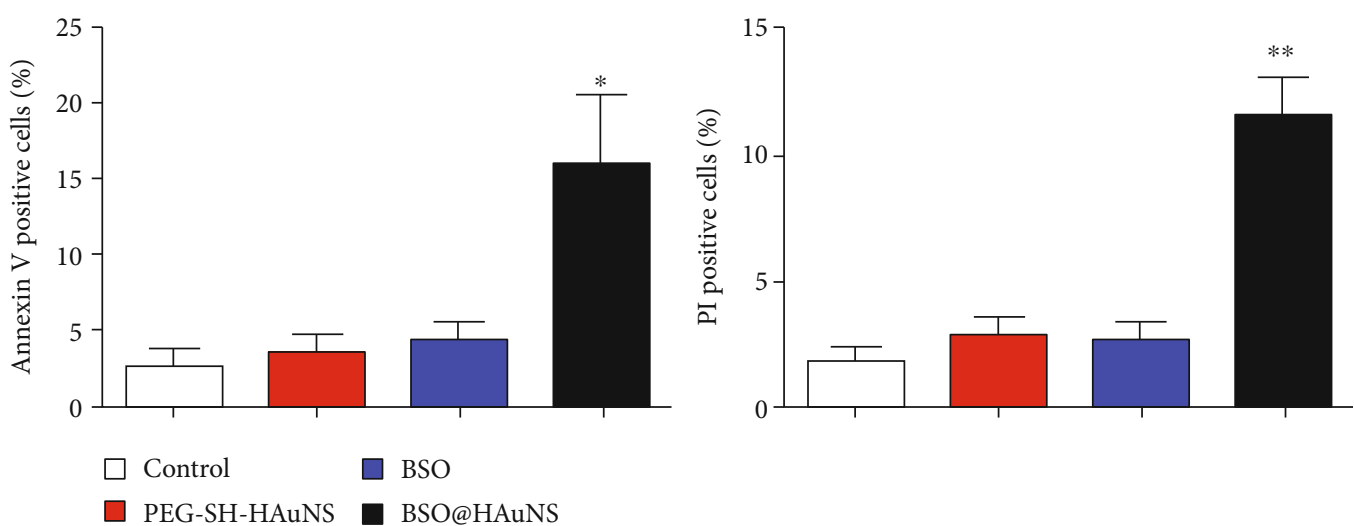

(b)

FIGURE 5: Effect of BSO@HAuNS on apoptosis and necrosis in A549 cells. (a) A549 cells were treated with BSO@HAuNS for 72 h, then harvested and washed twice with PBS, and stained with Annexin V-FITC and PI. The stained cells were measured with flow cytometry. (b) The percent of Annexin V positive or PI positive cells in each sample. Each bar represents the mean $( \pm S D, n=3) .{ }^{*} p<0.05$ and ${ }^{* *} p<0.01$ versus control. 


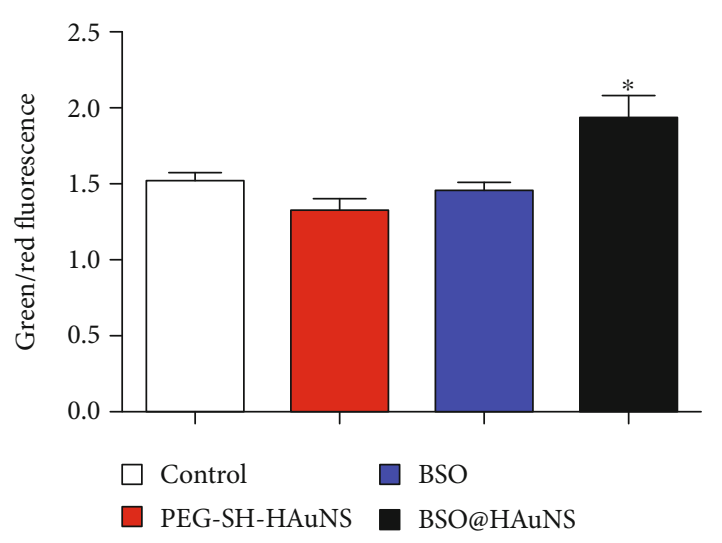

(a)

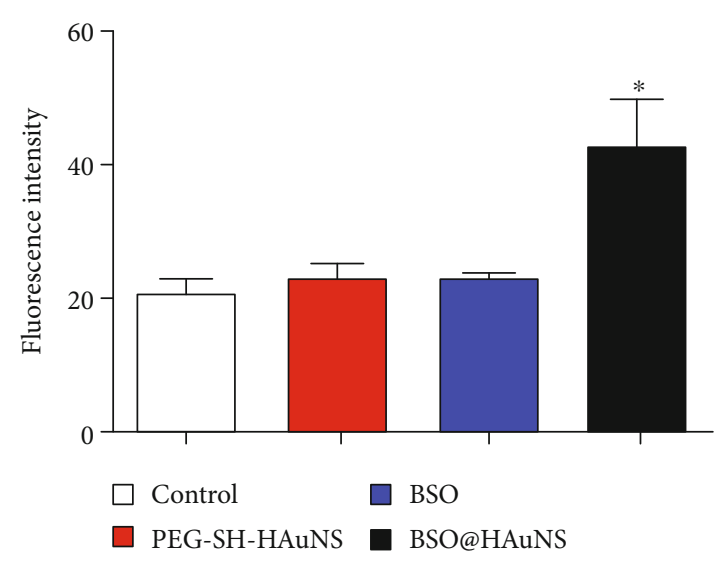

(b)

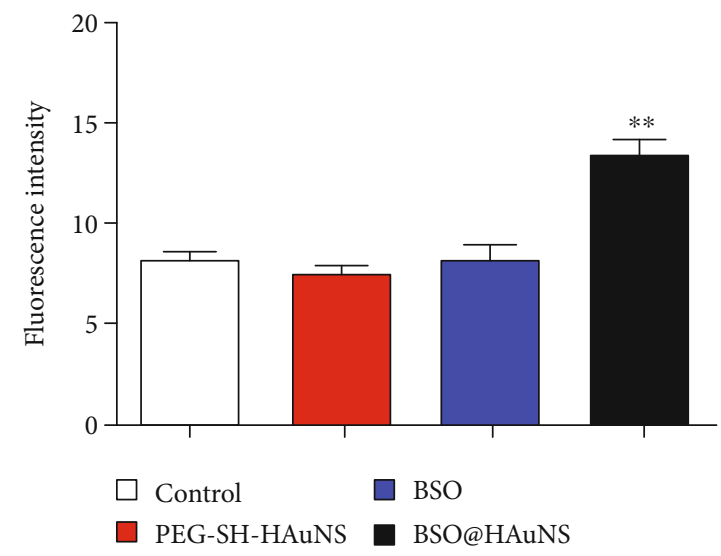

(c)

FIGURE 6: BSO@HAuNS induce cell death in mitochondrial-dependent pathway. A549 cells were exposed to various treatments for $72 \mathrm{~h}$, then harvested, and washed twice with PBS. (a) After treatment, the cells were processed by JC-1 staining and measured by flow cytometry. Mitochondrial membrane potential changes were quantified by fluorescence shift (red to green) of samples. (b) Following exposure to BSO, PEG-SH-HAuNS, and BSO@HAuNS, the Fluo-4-dependent fluorescence of the different samples was indicated by flow cytometry. Numbers depict the effect of BSO@HAuNS on A549 cytosolic $\mathrm{Ca}^{2+}$ concentration. Data shown are means $( \pm \mathrm{SD}, n=3)$. (c) Caspase-3 activity after BSO-loaded PEG-SH-HAuNS treatment for $72 \mathrm{~h}$ was determined with cleaved caspase-3 (Asp175) antibody (Alexa fluor 488 conjugate) by flow cytometry. ${ }^{*} p<0.05$ and ${ }^{* *} p<0.01$ compared with control.

the exposure of A549 cells to BSO@HAuNS was followed by a significant increase of Fluo4 fluorescence intensity pointing to an increase of cytosolic $\mathrm{Ca}^{2+}$.

3.8. BSO@HAuNS Induce Activation of Caspase-3 in A549 Cells. Because, mitochondrial-dependent apoptosis is initiated by recruitment and activation of caspases, further evidence for induction of the mitochondrial apoptotic pathway by BSO@HAuNS was provided by flow cytometry analysis of cleaved caspase-3 level. The expression of activated caspase-3 was found to significantly increase when A549 cells were treated with BSO@HAuNS than that treated with BSO and PEG-SH-HAuNS alone (Figure 6(c)). These results indicated that BSO@HAuNS-induced apoptosis is triggered by the caspase- 3 .

\section{Conclusions}

Striking a favorable balance between efficacy and toxicity is a significant challenge for the development of new cancer therapies. Researchers are making an effort to exploit an ideal agent that can enhance the selectivity and efficacy of anticancer agents and reduce their side effects to improve treatment for cancer patients. Gold nanoparticles (GNPs), as a unique drug delivery vehicle, have been attempted to achieve this goal. With their high stability, low toxicity, and excellent biocompatibility, gold nanoparticles hold great promise in biomedical application for treating inoperable cancers. A previous study by Libutti et al. reported a covalently linked PEGylated colloidal gold nanoparticle carrying recombinant human tumor necrosis factor alpha (rhTNF) that can selectively traffic to tumor tissue, deliver a therapeutic dose of rhTNF, and cause massive vascular leak and was safer and more effective than rhTNF [28]. Horo [29] investigated a novel silk coated materials showed effective drug delivery and sustained drug release, which demonstrated a maximum dose-dependent decrease in cell viability for the cells treated with folate conjugated silk fibroin-coated nanoparticles in Hela cell lines. Statistically significant data show that in in vitro conditions, cytostatic drugs attached to GNPs 
are more efficient than the classic anticancer drugs. This may be partially due to the increased uptake of the drugs by the tumor cells, since the unique pathophysiologic characteristics of tumor vessels enable macromolecules, including gold nanoparticles, to selectively accumulate in tumor tissues [30]. In addition, gold nanoparticles have a greater chance of reaching the targeted tumor tissues because of their proper size and the ability to circulate for longer times in the bloodstream, which constitutes an important mechanism that gold nanoparticles can selectively accumulate in the tumor site $[30,31]$. In this study, we present the synthesis and characterization of hollow gold nanoparticles functionalized as a drug delivery system. PEG was used for the modification of HAuNS' surface to enhance their biocompatibility.

Recently, gold nanoparticles also have been gaining fame as anticancer agents. Selim and Hendi showed that gold nanoparticles induce apoptosis in MCF-7 human breast cancer cells [32]. Gong et al. [33] reported that carbondot-supported atomically dispersed gold as a mitochondrial oxidative stress amplifier for cancer treatment various attempts to incorporate gold nanoparticles into cancer treatments have been made, with positive outcomes. Tiopronin was found to be nontoxic; however, gold nanoparticles coated with tiopronin (AuNPs-TP) display significantly cytotoxicity in HeLa cells. Oxidative stress was attributed to the cytotoxic effect of the AuNPs-TP [34]. In our previous work, we found that GNPs showed evident cytotoxicity in human lung cancer cells with low intracellular glutathione $[16,17]$. These results provided clear evidence that GNPs may be employed not only as carriers themselves but also as potential therapeutics by exploiting their capability to decrease intracellular GSH expression and generate cytotoxic responses. L-buthionine-sulfoximine (BSO) is known to deplete the intracellular pool of glutathione $[17,35]$. In this study, we evaluated the property of BSO@HAuNS as a novel nanomedicine for the therapy of human lung cancer.

In our results, in vitro cytotoxicity assay and Annexin V/PI flow cytometry measurements of the apoptotic and necrotic response of tumor cells to the BSO@HAuNS revealed the toxicity for the regimen combining. Oxidative stress has been shown to occur in cells following exposure to gold nanoparticles. ROS have been reported to damage the subcellular components and so cause cell death. Cellular responses to ROS depend on the cell's redox potential, which is mainly determined by the intracellular amount of GSH [25]. BSO@HAuNS produced a dramatic loss of intracellular GSH levels in A549 cells. So, the increase in ROS following BSO@HAuNS treatment explains the toxicity observed in human lung cancer cells. Furthermore, antioxidants (GSH) effectively inhibited ROS and reduced the cytotoxicity of the BSO@HAuNS. BSO@HAuNS were determined to be able to activate caspase signaling by altering the ratio of cytosolic $\mathrm{Ca}^{2+}$ as well as mitochondrial membrane integrity. We also provide indirect evidence that the inhibition of tumor cells byBSO@HAuNS is achieved via the mechanism of cell apoptosis and necrosis. This is consistent with previous results showing that antioxidants could protect lung cancer cells from cytotoxicity of GNPs [16, 17]. This novel nanomedicine offers a different approach to cancer therapy and holds significant potential to improve disease management and treatment of cancer in the future. Following studies will focus on evaluating the in vivo distribution and toxicity of the BSO@HAuNS at the whole body tissue. Otherwise, it is reported that cancer-specific conditions such as acidic $\mathrm{pH}$ and higher levels of glutathione, heat, or light can be used to trigger the release of the drug from the nanocarrier [36]. These parameters act as stimuli for the smart release of the drug $[36,37]$. However, power evidences are needed to further clarify whether "BSO is released from BSO@HAuNS." Therefore, in the next step, we will illustrate the specific characteristics which can trigger drug release (i.e., $\mathrm{pH}$, glutathione, temperature, and light).

\section{Data Availability}

The data used to support the findings of this study are included within the article.

\section{Conflicts of Interest}

The authors declare that there are no conflicts of interest.

\section{Acknowledgments}

This study was supported by the National Natural Science Foundation of China (grant no. 81803635). The authors thank Dr. Xiaohu Gu and Professor Yi Ding from Shandong University School of Chemistry and Chemical Engineering, for supporting the hollow gold nanoparticles.

\section{References}

[1] J. Ferlay, M. Colombet, I. Soerjomataram et al., "Estimating the global cancer incidence and mortality in 2018: GLOBOCAN sources and methods," International Journal of Cancer, vol. 144, no. 8, pp. 1941-1953, 2019.

[2] K. Sztandera, M. Gorzkiewicz, and B. Klajnert-Maculewicz, "Gold nanoparticles in cancer treatment," Molecular Pharmaceutics, vol. 16, no. 1, pp. 1-23, 2019.

[3] Sánchez-Paradinas, Pérez-Andrés, Almendral-Parra et al., "Enhanced cytotoxic activity of bile acid cisplatin derivatives by conjugation with gold nanoparticles," Journal of Inorganic Biochemistry, vol. 131, pp. 8-11, 2014.

[4] A. Kumar, H. Ma, X. Zhang et al., "Gold nanoparticles functionalized with therapeutic and targeted peptides for cancer treatment," Biomaterials, vol. 33, no. 4, pp. 1180-1189, 2012.

[5] A. Assali, O. Akhavan, M. Adeli et al., "Multifunctional coreshell nanoplatforms (gold@graphene oxide) with mediated NIR thermal therapy to promote miRNA delivery," Nanomedicine, vol. 14, no. 6, pp. 1891-1903, 2018.

[6] A. Mioc, M. Mioc, R. Ghiulai et al., "Gold nanoparticles as targeted delivery systems and theranostic agents in cancer therapy," Current Medicinal Chemistry, vol. 26, no. 35, pp. 6493-6513, 2019.

[7] U. A. Ashfaq, M. Riaz, E. Yasmeen, and M. Z. Yousaf, "Recent advances in nanoparticle-based targeted drug-delivery systems against cancer and role of tumor microenvironment," Critical Reviews in Therapeutic Drug Carrier Systems, vol. 34, no. 4, pp. 317-353, 2017. 
[8] K. Kalimuthu, B. C. Lubin, A. Bazylevich et al., "Gold nanoparticles stabilize peptide-drug-conjugates for sustained targeted drug delivery to cancer cells," Journal of Nanobiotechnology, vol. 16, no. 1, p. 34, 2018.

[9] M. Khan, S. Boumati, C. Arib et al., "Doxorubicin (DOX) gadolinium-gold-complex: a new way to tune hybrid nanorods as theranostic agent," International Journal of Nanomedicine, vol. Volume 16, pp. 2219-2236, 2021.

[10] Z. Arab-Bafrani, D. Shahbazi-Gahrouei, and M. Abbasian, "Rapid delivery of gold nanoparticles into colon cancer HT29 cells by electroporation: in-vitro study," Journal of Biomedical Physics and Engineering, vol. 10, no. 2, pp. 161-166, 2012.

[11] A. R. Fernandes, J. Jesus, P. Martins et al., "Multifunctional gold-nanoparticles: a nanovectorization tool for the targeted delivery of novel chemotherapeutic agents," Journal of Controlled Release, vol. 245, pp. 52-61, 2017.

[12] A. Kumar, S. Huo, X. Zhang et al., "Neuropilin-1-targeted gold nanoparticles enhance therapeutic efficacy of platinum(IV) drug for prostate cancer treatment," ACS Nano, vol. 8, no. 5, pp. 4205-4220, 2014.

[13] N. A. Koonce, C. M. Quick, M. E. Hardee et al., "Combination of Gold Nanoparticle-Conjugated Tumor Necrosis Factor- $\alpha$ and Radiation Therapy Results in a Synergistic Antitumor Response in Murine Carcinoma Models," International Journal of Radiation Oncology • Biology • Physics, vol. 93, no. 3, pp. 588-596, 2015.

[14] D. N. Heo, D. H. Yang, H. J. Moon et al., "Gold nanoparticles surface-functionalized with paclitaxel drug and biotin receptor as theranostic agents for cancer therapy," Biomaterials, vol. 33, no. 3, pp. 856-866, 2012.

[15] D. Mateo, P. Morales, A. Ávalos, and A. I. Haza, "Oxidative stress contributes to gold nanoparticle-induced cytotoxicity in human tumor cells," Toxicology Mechanisms and Methods, vol. 24, no. 3, pp. 161-172, 2014.

[16] T. Yuan, J. Sun, J. Tian, J. Hu, H. Yin, and J. Yin, "Involvement of $\mathrm{ABC}$ transporters in the detoxification of non-substrate nanoparticles in lung and cervical cancer cells," Journal of Physical Chemistry C, vol. 455, no. 26, article 152762, 2021.

[17] M. Liu, X. H. Gu, K. Zhang et al., "Gold nanoparticles trigger apoptosis and necrosis in lung cancer cells with low intracellular glutathione," Journal of Nanoparticle Research, vol. 15, no. 8, p. 1745, 2013.

[18] J. You, J.Zhou, M. Zhou et al., "Pharmacokinetics, clearance, and biosafety of polyethylene glycol-coated hollow gold nanospheres," Particle and Fibre Toxicology, vol. 11, no. 1, p. 26, 2014.

[19] R. Jin, J. Yang, D. Zhao et al., "Hollow gold nanoshellsincorporated injectable genetically engineered hydrogel for sustained chemo-photothermal therapy of tumor," Journal of Nanobiotechnology, vol. 17, no. 1, p. 99, 2019.

[20] D. L. Meng, L. Shang, X. H. Feng, X. F. Huang, and X. Che, "Xanthoceraside hollow gold nanoparticles, green pharmaceutics preparation for poorly water-soluble natural anti-AD medicine," International Journal of Pharmaceutics, vol. 506, no. 1-2, pp. 184-190, 2016.

[21] F. Hu, Y. Zhang, G. Chen, C. Li, and Q. Wang, "Double-walled $\mathrm{Au}$ nanocage $/ \mathrm{SiO}_{2}$ nanorattles: integrating SERS imaging, drug delivery and photothermal therapy," Small, vol. 11, no. 8, pp. 985-993, 2015.

[22] H. M. Lee, D. H. Kim, H. L. Lee, B. Cha, D. H. Kang, and Y. I. Jeong, "Synergistic effect of buthionine sulfoximine on the chlorin e6-based photodynamic treatment of cancer cells,"
Archives of Pharmacal Research, vol. 42, no. 11, pp. 990-999, 2019.

[23] A. R. Bonilla-Porras, M. Jimenez-Del-Rio, and C. Velez-Pardo, "N-acetyl-cysteine blunts 6-hydroxydopamine- and L-buthionine-sulfoximine-induced apoptosis in human mesenchymal stromal cells," Molecular Biology Reports, vol. 46, no. 4, pp. 4423-4435, 2019.

[24] A. M. Alkilany and C. J. Murphy, "Toxicity and cellular uptake of gold nanoparticles: what we have learned so far?," Journal of Nanoparticle Research, vol. 12, no. 7, pp. 2313-2333, 2010.

[25] A. Abdal Dayem, M. K. Hossain, S. B. Lee et al., "The role of reactive oxygen species (ROS) in the biological activities of metallic nanoparticles," International Journal of Molecular Sciences, vol. 18, no. 1, p. 120, 2017.

[26] Y. L. Wang, H. F. Liu, X. J. Shi, and Y. Wang, “Antiproliferative activity of Farnesol in HeLa cervical cancer cells is mediated via apoptosis induction, loss of mitochondrial membrane potential (lambdapsim) and PI3K/Akt signalling pathway," Journal of the Balkan Union of Oncology, vol. 23, no. 3, pp. 752-757, 2018.

[27] A. Rasola and P. Bernardi, "Mitochondrial permeability transition in $\mathrm{Ca}(2+)$-dependent apoptosis and necrosis," Cell Calcium, vol. 50, no. 3, pp. 222-233, 2011.

[28] S. K. Libutti, G. F. Paciotti, A. A. Byrnes et al., "Phase I and pharmacokinetic studies of CYT-6091, a novel PEGylated colloidal gold-rhTNF nanomedicine," Clinical Cancer Research, vol. 16, no. 24, pp. 6139-6149, 2010.

[29] H. Horo, S. Bhattacharyya, B. Mandal, and L. M. Kundu, "Synthesis of functionalized silk-coated chitosan-gold nanoparticles and microparticles for target-directed delivery of antitumor agents," Carbohydrate Polymers, vol. 258, p. 117659, 2021.

[30] J. Liu, M. Yu, C. Zhou, S. Yang, X. Ning, and J. Zheng, "Passive tumor targeting of renal-clearable luminescent gold nanoparticles: long tumor retention and fast normal tissue clearance," Journal of the American Chemical Society, vol. 135, no. 13, pp. 4978-4981, 2013.

[31] P. Kumari, B. Ghosh, and S. Biswas, "Nanocarriers for cancertargeted drug delivery," Journal of Drug Targeting, vol. 24, no. 3, pp. 179-191, 2016.

[32] M. E. Selim and A. A. Hendi, "Gold nanoparticles induce apoptosis in MCF-7 human breast cancer cells," Asian Pacific Journal of Cancer Prevention, vol. 13, no. 4, pp. 1617-1620, 2012.

[33] N. Gong, X. Ma, X. Ye et al., "Carbon-dot-supported atomically dispersed gold as a mitochondrial oxidative stress amplifier for cancer treatment," Nature Nanotechnology, vol. 14, no. 4, pp. 379-387, 2019.

[34] L. Cui, P. Zahedi, J. Saraceno, R. Bristow, D. Jaffray, and C. Allen, "Neoplastic cell response to tiopronin-coated gold nanoparticles," Nanomedicine, vol. 9, no. 2, pp. 264-273, 2013.

[35] Y. Sun, Y. Zheng, C. Wang, and Y. Liu, "Glutathione depletion induces ferroptosis, autophagy, and premature cell senescence in retinal pigment epithelial cells," Cell Death \& Disease, vol. 9, no. 7, p. 753, 2018.

[36] J. C. Imperiale, G. B. Acosta, and A. Sosnik, "Polymer-based carriers for ophthalmic drug delivery," Journal of Controlled Release, vol. 285, pp. 106-141, 2018.

[37] S. A. R. Kazmi, M. Z. Qureshi, S. Ali, and J. F. Masson, "In vitro drug release and biocatalysis from $\mathrm{pH}$-responsive gold nanoparticles synthesized using doxycycline," Langmuir, vol. 35, no. 49, pp. 16266-16274, 2019. 